\title{
Probing the nature and origin of dust in the reddened quasar IC 4329A with global modelling from X-ray to infrared
}

\author{
Missagh Mehdipour and Elisa Costantini
}

\begin{abstract}
SRON Netherlands Institute for Space Research, Sorbonnelaan 2, 3584 CA Utrecht, The Netherlands e-mail: m.mehdipour@sron.nl
\end{abstract}

Received 24 June 2018 / Accepted 13 August 2018

\begin{abstract}
Cosmic dust is a key tracer of structure formation and evolution in the universe. In active galactic nuclei (AGN) the origin and role of dust are uncertain. Here, we have studied dust in the X-ray bright and reddened type-1 quasar IC 4329A, which exhibits an ionised AGN wind. We incorporated high-resolution X-ray and mid-IR spectroscopy, combined with broad-band continuum modelling, to investigate the properties of dust in this AGN. We used new Chandra HETGS observations taken in June 2017, as well as archival data from XMM-Newton, Swift, HST, Spitzer, IRAS, and Herschel for our IR-optical-UV-X-ray modelling. Two distinct components of dust in IC 4329A are found. One component is in the interstellar medium (ISM) of the host galaxy, and the other is a nuclear component in the AGN torus and its associated wind. The emitting dust in the torus is evident in mid-IR emission $(9.7$ and $18 \mu \mathrm{m}$ features), while dust in the wind is present through both reddening and $\mathrm{X}$-ray absorption $(\mathrm{O}, \mathrm{Si}$, and $\mathrm{Fe}$ edge features). The gas depletion factors into dust for $\mathrm{O}, \mathrm{Si}$, and $\mathrm{Fe}$ are measured. We derive an intrinsic reddening $E(B-V) \approx 1.0$, which is most consistent with a grey (flat) extinction law. The AGN wind consists of three ionisation components. From analysis of long-term changes in the wind, we determine limits on the location of the wind components. The two lowest ionisation components are likely carriers of dust from the AGN torus. We find that the dust in the nuclear component of IC 4329A is different from dust in the Milky Way. The dust grains in the AGN torus and wind are likely larger than the standard Galactic dust, and are in a porous composite form (containing amorphous silicate with iron and oxygen). This can be a consequence of grain coagulation in the dense nuclear environment of the AGN.
\end{abstract}

Key words. X-rays: galaxies - galaxies: active - techniques: spectroscopic - dust, extinction

\section{Introduction}

Cosmic dust is widespread in the universe. It can be used to trace the evolutionary paths of planets, stars, and even black holes. Dust grains in the ISM of galaxies alter the appearance of the observable universe through absorption and scattering of photons. Thus, determining the effects of dust is needed for studying a wide range of astrophysical phenomena. Indeed a significant fraction of ISM is locked up in dust grains (e.g. Jenkins 2009). However, many aspects of cosmic dust remain poorly understood, such as: their chemical composition and origin; their physical properties and spectral signatures; their formation and evolution, and destruction mechanisms; and their role and impact on their environment. In active galactic nuclei (AGN) the properties of dust are particularly uncertain (see e.g. the review by Li 2007). Also, dust in AGN can be associated to different possible origins, such as: dust lanes of recent galaxy merger remnants, or dusty winds from the AGN (see e.g. Komossa \& Fink 1997; Reynolds et al. 1997; Lee et al. 2001; Crenshaw \& Kraemer 2001).

Dust is an essential player in the unification theory of AGN, where the supermassive black hole $(\mathrm{SMBH})$ and the accretion disk are surrounded by an optically-thick dusty torus. The observational properties of AGN are hence strongly influenced by our viewing angle relative to the orientation of this obscuring torus (Antonucci \& Miller 1985; Antonucci 1993; Urry \& Padovani 1995). Yet, our knowledge of dust properties in the AGN torus and its environment is limited.
Accretion onto SMBHs at the core of AGN is accompanied by winds of gas, which couple the SMBHs to their environment. The observed relations between SMBHs and their host galaxies, such as the $\mathrm{M}-\sigma$ relation (Ferrarese \& Merritt 2000), indicate that SMBHs and their host galaxies are likely co-evolved through a feedback mechanism. The AGN winds can play a key role in this co-evolution as they can significantly impact star formation (e.g. Silk \& Rees 1998) and chemical enrichment of the surrounding intergalactic medium (e.g. Oppenheimer \& Davé 2006). However, there are significant gaps in our understanding of the outflow phenomenon in AGN, which cause major uncertainties in determining their role and impact in galaxy evolution.

The origin and physical structure of AGN winds are generally poorly understood. Different mechanisms have been postulated for the launch and driving of winds from either the accretion disk or the AGN torus (e.g. Krolik \& Kriss 2001; Proga \& Kallman 2004; Fukumura et al. 2010). However, their association to the different kinds of winds found from observations is uncertain. AGN winds can originate as either thermally-driven (Krolik \& Kriss 2001) or radiatively-driven (Dorodnitsyn et al. 2008) winds from the dusty torus. Indeed the warm-absorber winds, which are commonly detected in the UV and X-ray spectra of bright AGN, are most consistent with being torus winds (e.g. Kaastra et al. 2012; Mehdipour et al. 2018); thus dust could be mixed with such winds. Importantly, the infrared (IR) radiation pressure on dust grains can boost winds from the torus (Dorodnitsyn et al. 2011). Therefore, establishing the existence of dust in AGN winds is important for 
understanding the driving mechanism of AGN winds, and defining their impact on their environment. This is needed for assessing the contribution of such winds to AGN feedback. There are observational evidence, which indicate that AGN winds are likely carriers of dust into the ISM. For example, in the case of ESO 113-G010 (Mehdipour et al. 2012), presence of dust embedded in the AGN wind was inferred based on the altering of the AGN emission, dust-to-gas ratio arguments, and the properties of the wind.

In order to advance our understanding of cosmic dust in the universe we need to utilise all available tools at our disposal. The X-ray energy band, which is invaluable for exploring both the cold and hot gas, is a relatively new scientific window for dust studies. The X-ray absorption fine structures (XAFS) at the $\mathrm{K}$ edge of $\mathrm{O}, \mathrm{Mg}, \mathrm{Si}, \mathrm{Fe}$, and the LII and LIII edges of Fe provide distinct and unblended signatures of dust grains in X-rays (e.g. Lee et al. 2009; Costantini et al. 2012; Zeegers et al. 2017; Rogantini et al. 2018). Therefore, in addition to the traditional low-energy domain observations, like in the IR, the X-ray band enables us to directly access the chemical composition of dust in the diffuse ISM of galaxies. Thus, high-resolution X-ray spectroscopy provides a powerful and sensitive diagnostic tool to probe the properties of both gas and dust. This is invaluable for understanding the formation and evolution history of galaxies, including the host galaxies of AGN. Indeed X-ray spectroscopy can help in studying the chemistry of dust in AGN (e.g. Lee et al. 2013), which is an important indicator of the evolutionary phase of AGN.

IC 4329A is bright nearby AGN at redshift $z=0.016054$ (Willmer et al. 1991). It has been described as "an extreme Seyfert galaxy" (Disney 1973) and "the nearest quasar" (Wilson \& Penston 1979), based on the spectroscopy of its broad optical emission lines. However, it is technically classified as a Seyfert 1.2 by Véron-Cetty \& Véron (2006). The host galaxy of the AGN is highly inclined (i.e. edge-on), with the observed ratio of minor to major axis $b / a=0.28$ (de Vaucouleurs et al. 1991). A prominent dust lane bisects the nucleus of IC 4329A, which can be seen in the HST image of Fig. 1. The dust lane is indicative of the past merger history of this galaxy. IC 4329A is a member of a group of seven galaxies (Kollatschny \& Fricke 1989). It is displaced by a projected distance of $59 \mathrm{kpc}$ from the giant lenticular galaxy IC 4329 (Wolstencroft et al. 1995). The relative orientations of the axes of the AGN and the disk of the host galaxy may have been influenced by interaction between IC 4329A and its massive neighbour IC 4329 (Wolstencroft et al. 1995).

The mass of the supermassive black hole $\left(M_{\mathrm{BH}}\right)$ in IC 4329A is not accurately determined. The $M_{\mathrm{BH}}$ from reverberation study is poorly constrained due to low quality lightcurves, and hence unreliable lag measurements, from which Peterson et al. (2004) estimate an upper limit of $\sim 3 \times 10^{7} M_{\odot}$. However, measurements using other methods find higher $M_{\mathrm{BH}}$. Using the empirical relation derived by McHardy et al. (2006) between the bolometric luminosity $L_{\mathrm{bol}}, M_{\mathrm{BH}}$, and the break frequency in the X-ray power spectral density function (PSD), Markowitz (2009) calculate $M_{\mathrm{BH}}=1.3_{-0.3}^{+1.0} \times 10^{8} M_{\odot}$. Also, using relations between $M_{\mathrm{BH}}$ and the stellar velocity dispersion $\sigma_{*}$, Markowitz (2009) find $M_{\mathrm{BH}} \approx 2_{-1}^{+2} \times 10^{8} M_{\odot}$. Furthermore, de La Calle Pérez et al. (2010) report $M_{\mathrm{BH}} \sim 1.2 \times 10^{8} M_{\odot}$. From the AGN sample study of Vasudevan et al. (2010), the black hole mass is estimated from the $K$-band luminosity of the host galaxy bulge, which in the case of IC 4329A is found to be about $2 \times 10^{8} M_{\odot}$. Therefore, in our calculations we assume $M_{\mathrm{BH}}$ is $\sim 1-2 \times 10^{8} M_{\odot}$.

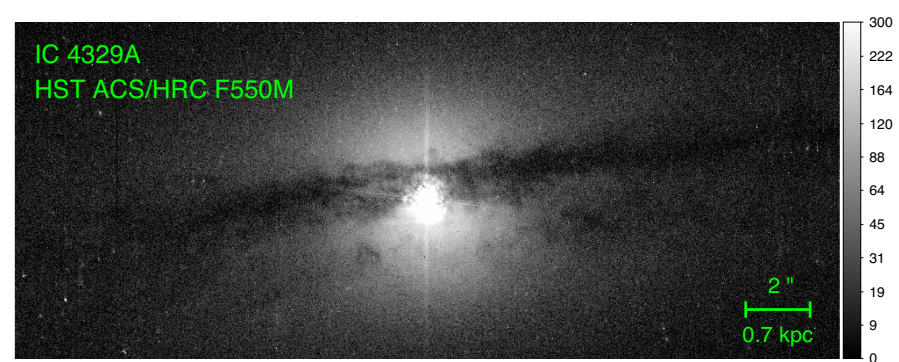

Fig. 1. Nuclear region of IC 4329A as observed by the HST, showing the presence of a dust lane covering the nucleus. The image is obtained from an observation taken with the ACS/HRC F550M filter on 22 February 2006, and is displayed with a logarithmic intensity scale.

Steenbrugge et al. (2005) studied the X-ray absorption in IC 4329A with XMM-Newton RGS spectroscopy. They found absorption by neutral gas in the host galaxy of the AGN, as well as warm absorption by an AGN wind. Despite significant absorption, IC 4329A is bright enough for high-resolution X-ray spectroscopy, which is often not the case for many reddened and absorbed AGN, making IC 4329A a valuable target. The column density $N_{\mathrm{H}}$ of the neutral gas was measured to be about $1.7 \times 10^{21} \mathrm{~cm}^{-2}$ (Steenbrugge et al. 2005). The warm absorber was found to have four ionisation components, with a total $N_{\mathrm{H}}$ of about $1.0 \times 10^{22} \mathrm{~cm}^{-2}$. The velocity of the warm absorber components ranges from $-200 \pm 100$ to $+20 \pm 160 \mathrm{~km} \mathrm{~s}^{-1}$. Possible X-ray spectral features of dust were not investigated in Steenbrugge et al. (2005).

Dust reddened and absorbed AGN are often too faint for dust X-ray spectroscopy because of strong absorption. Therefore, finding a suitable target is key. For our X-ray spectroscopic investigation of dust in winds, the AGN target must meet these required selection criteria: (1) bright enough in X-rays; (2) displays significant intrinsic reddening; (3) exhibits evidence of dust features in both X-rays and IR; (4) shows the presence of an AGN wind; (5) minimum amount of reddening and absorption contamination by the Milky Way in our of sight. Among many candidates that we systematically searched, IC 4329A is one of the most suitable AGN that meets all these criteria. Our newly acquired Chandra-HETGS observations of IC 4329A are presented for the first time in this paper.

The structure of the paper is as follows. The observations and the reduction of data are described in Sect. 2. The modelling of the spectral energy distribution (SED) is presented in Sect. 3. The modelling of the X-ray absorption by the ISM gas and the AGN wind are explained in Sect. 4. The multi-wavelength analysis of dust in IC 4329A based on reddening, IR emission features, and X-ray absorption features, is presented in Sect. 5. We discuss all our findings in Sect. 6, and give concluding remarks in Sect. 7.

The spectral analysis and modelling presented in this paper were done using the SPEX package (Kaastra et al. 1996) v3.04.00. The spectra shown in this paper are backgroundsubtracted and are displayed in the observed frame. We use C-statistics for spectral fitting and give errors at the $1 \sigma$ confidence level. We adopt a luminosity distance of 69.61 Mpc in our calculations with the cosmological parameters $H_{0}=70 \mathrm{~km} \mathrm{~s}^{-1} \mathrm{Mpc}^{-1}, \Omega_{\Lambda}=0.70$, and $\Omega_{\mathrm{m}}=0.30$. We assume proto-solar abundances of Lodders et al. (2009) in all our computations in this paper. 


\section{Observations and data processing}

The observation log of data used in our spectral analysis are provided in Table 1 . We describe the processing of data from different observatories in the following.

\subsection{X-ray data}

All Chandra observations of IC 4329A have been taken with HETGS (Canizares et al. 2005). In all the HETGS observations, the ACIS camera was operated in the timed exposure (TE) read mode and the faint data mode. The data were reduced using the Chandra Interactive Analysis of Observations (CIAO, Fruscione et al. 2006) v4.9 software and the calibration database (CALDB) v4.7.3. The chandra_repro script of CIAO and its associated tools were used for the reduction of the data and production of the final grating products (PHA2 spectra, RMF and ARF response matrices). The MEG and HEG +/- firstorder spectra and their response matrices were combined using the CIAO combine_grating_spectra script. The short-term X-ray variability, seen by the HETGS observations on days timescale, correspond to about $10 \%$ flux variation around the mean. This is a small variability, allowing us to stack the individual spectra in order to enhance the signal-to-noise ratio. The fitted spectral range is $2.5-26 \AA$ for MEG, and 1.55-14.5 $\AA$ for HEG. Over these energy bands, the HEG/MEG flux ratio is nearly constant at 0.952 . We take into account this instrumental flux difference between HEG and MEG by re-scaling the normalisation of HEG relative to MEG in our spectral modelling. The low and high energy data outside of these ranges are ignored because of deviations in the HEG/MEG flux ratio caused by the increasing calibration uncertainties of the instruments.

The XMM-Newton data were processed using the Science Analysis System (SAS v16.0.0). The RGS (den Herder et al. 2001) instruments were operated in the standard Spectro+Q mode for the XMM-Newton observations of IC 4329A (Table 1). The data were processed through the rgsproc pipeline task; the source and background spectra were extracted and the response matrices were generated. We filtered out time intervals with background count rates $>0.1$ count s$^{-1}$ in CCD number 9 . The rgscombine task was used to stack the RGS first-order spectra. Our fitted spectral range is 7-35 $\AA$ for RGS. The EPIC-pn instrument (Strüder et al. 2001) was operated in the Full-Frame mode with the Medium Filter during the 2001 observation, and in the Small-Window mode with the Thin Filter during the 2003 observation. Periods of high flaring background for EPIC-pn (exceeding 0.4 count $\mathrm{s}^{-1}$ ) were filtered out by applying the \#XMMEA_EP filtering. The EPIC-pn spectra were extracted from a circular region centred on the source with a radius of $40^{\prime \prime}$. The background was extracted from a nearby source-free region of radius $40^{\prime \prime}$ on the same CCD as the source. The pileup was evaluated to be small at about $2 \%$. The single and double events were selected for the EPIC-pn (PATTERN <= 4). Response matrices were generated for the spectrum of each observation using the rmfgen and arfgen tasks. The epicspeccombine task was used for stacking the EPIC-pn spectra. Our fitted spectral range for EPIC-pn is from $1.38 \mathrm{keV}(9 \AA)$ to $10 \mathrm{keV}$, since the soft $\mathrm{X}$-ray band $(0.35-1.8 \mathrm{keV})$ is simultaneously modelled with RGS. The EPIC-pn/RGS flux ratio at the overlapping energy band is found to be 0.91 , which we take into account by re-scaling in our simultaneous fitting of the RGS and EPIC-pn spectra. In our spectral modelling we first derived a time-averaged model fitted to the stacked spectra from all available observations, and then applied this model to determine the variability in the X-ray continuum and
Table 1. Observation log of the data used in our spectral modelling.

\begin{tabular}{lccc}
\hline \hline Observatory & Obs. ID & $\begin{array}{c}\text { Obs. date } \\
\text { yyyy-mm-dd }\end{array}$ & $\begin{array}{c}\text { Length } \\
(\mathrm{ks})\end{array}$ \\
\hline Chandra/HETGS & 2177 & $2001-08-26$ & 59.1 \\
Chandra/HETGS & 20070 & $2017-06-06$ & 91.8 \\
Chandra/HETGS & 19744 & $2017-06-12$ & 12.4 \\
Chandra/HETGS & 20095 & $2017-06-13$ & 33.3 \\
Chandra/HETGS & 20096 & $2017-06-14$ & 19.8 \\
Chandra/HETGS & 20097 & $2017-06-17$ & 16.8 \\
\hline XMM-Newton & 0101040401 & $2001-01-31$ & 13.9 \\
XMM-Newton & 0147440101 & $2003-08-06$ & 136.0 \\
\hline Swift/UVOT & 00033058001 & $2013-12-21$ & 1.9 \\
\hline HST/WFPC2/F814W & U5GU0401R & $2000-02-25$ & 0.012 \\
HST/NICMOS/F160W & N4JQ06010 & $1998-05-21$ & 0.22 \\
HST/NICMOS/F196N & N4JQ06070 & $1998-05-21$ & 0.51 \\
HST/NICMOS/F200N & N4JQ060A0 & $1998-05-21$ & 0.90 \\
HST/NICMOS/F222M & N4JQ06040 & $1998-05-21$ & 0.26 \\
\hline Spitzer/IRAC & 12472576 & $2005-07-18$ & 0.047 \\
Spitzer/IRAC & 18038784 & $2006-08-13$ & 1.3 \\
Spitzer/MIPS & 10642176 & $2006-02-15$ & 0.53 \\
Spitzer/IRS & 4848640 & $2004-07-13$ & 1.3 \\
Spitzer/IRS & 18506496 & $2007-07-29$ & 0.96 \\
\hline Herschel/PACS/70- $\mu \mathrm{m}$ & 1342236918 & $2012-01-07$ & 0.052 \\
Herschel/PACS/160- $\mu \mathrm{m}$ & 1342236919 & $2012-01-07$ & 0.052 \\
Herschel/SPIRE & 1342236198 & $2012-01-02$ & 0.169 \\
\hline
\end{tabular}

Notes. The dates correspond to the start time of the observations in UTC. In addition to the above data, we also use fluxes at 60 and $100 \mu \mathrm{m}$ from the IRAS survey observations.

the X-ray absorption between the 2003 and 2017 epochs, when the deepest X-ray observations were taken.

\subsection{Optical and UV data}

In order to determine the optical and UV part of the SED, we made use of photometric data from XMM-Newton OM (Mason et al. 2001) and Swift UVOT (Roming et al. 2005). We use data taken with the $V, B, U$, UVW1, UVM2 and UVW2 filters. For a description of the reduction of OM and UVOT data, we refer to Appendix A in Mehdipour et al. (2015) and references therein, which applies to the data used here. The size of the circular aperture used for our photometry was set to a diameter of $12^{\prime \prime}$ for OM, and $10^{\prime \prime}$ for UVOT, which is the optimum aperture size based on the calibration of these instruments.

The continuum at the UV and optical, and lower energies, is approximated to be constant over time in our SED modelling. The optical and UV fluxes from the OM and UVOT are consistent with each other at the overlapping filters despite being taken years apart. Furthermore, from examining the long-term variability in the UVOT observations, we find that the flux variation in the $V$ band is about $4 \%$ around the mean. Therefore, the continuum at the optical and lower energies is not too variable for the purpose of approximating the SED by simultaneously fitting the individual observations given in Table 1.

\subsection{Infrared data}

To determine the SED spanning near-IR to far-IR energies, we have utilised data from HST, Spitzer, IRAS, and Herschel. The HST images from Wide Field and Planetary Camera 2 (WFPC2) F814W filter, and the Near Infrared Camera and Multi-Object Spectrometer (NICMOS) F160W, F196N, F200N, F222M 
filters, were retrieved from Mikulski Archive for Space Telescopes (MAST). We carried out aperture photometry with a diameter of $10^{\prime \prime}$ on the central source.

The Spitzer Multi-band Imaging Photometer (MIPS) spectrum was retrieved from the Spitzer Heritage Archive (SHA). The MIPS instrument, operating in the SED mode, provides a low-resolution spectrum from 53 to $100 \mu \mathrm{m}$. Furthermore, the Spitzer Infrared Array Camera (IRAC) photometric measurements at 3.6, 4.5, 5.8, 8.0 $\mu$ m were taken from the Spitzer study of a sample of AGN by Gallimore et al. (2010). The Spitzer Infrared Spectrograph (IRS) observations, operating in the IRS Stare mode, were used to extract the spectra. The IRS spectra from the low-resolution modules, short-low (SL) and longlow (LL), were retrieved from the Combined Atlas of Sources with Spitzer IRS Spectra (CASSIS). The spectra from the highresolution modules, short-high $(\mathrm{SH})$ and long-high $(\mathrm{LH})$, were processed through the $\mathrm{c} 2 \mathrm{~d}$ pipeline and optimal PSF extraction.

The Infrared Astronomical Satellite (IRAS) flux measurement at 60 and $100 \mu \mathrm{m}$ were obtained from the IRAS Faint Source catalogue v2.0 (Moshir 1990). We have also utilised far-infrared (FIR) data from Herschel PACS (70 and $160 \mu \mathrm{m}$ filters) and SPIRE (250, 350, and $500 \mu \mathrm{m}$ filters). The photometric flux measurements were obtained from the HerschelSwift/BAT AGN sample studies of Meléndez et al. (2014) and Shimizu et al. (2016), which report on the PACS and SPIRE observations, respectively.

\section{Determination of the spectral energy distribution}

Here we present our modelling of the components of the IRoptical-UV-X-ray continuum. We note that our continuum modelling is tied to the modelling of the X-ray absorption, reddening, and dust IR emission features, which are presented separately in later sections (Sects. 4 and 5).

\subsection{Primary IR-optical-UV-X-ray continuum of the AGN}

We started by applying a power-law component (pow) to fit the Xray spectrum of IC 4329A (Fig. 2). The X-ray spectrum is strongly absorbed, and the modelling of this absorption is described in Sect. 4. The X-ray power-law continuum model represents Compton up-scattering of the disk photons in an optically-thin and hot corona in IC 4329A. The high-energy exponential cut-off of the power-law was set to $186 \mathrm{keV}$ (Brenneman et al. 2014) based on NuSTAR and Suzaku observations. A low-energy exponential cut-off was also applied to the power-law continuum to prevent exceeding the energy of the seed disk photons. The photon index $\Gamma$ of the power-law is $\Gamma=1.78 \pm 0.01$.

In addition to the power-law, the soft X-ray continuum of IC 4329A includes a "soft X-ray excess" component (Cappi et al. 1996; Perola et al. 1999; Steenbrugge et al. 2005). To model the soft excess in IC 4329A, we use the broad-band model derived in Mehdipour et al. (2015) for NGC 5548, in which the soft excess is modelled by warm Comptonisation (see e.g. Magdziarz et al. 1998; Mehdipour et al. 2011; Done et al. 2012; Petrucci et al. 2013; Kubota \& Done 2018). In this explanation of the soft excess, the seed disk photons are up-scattered in an optically thick and warm corona to produce the soft X-ray excess. The comt model in SPEX produces a thermal opticalUV disk component modified by warm Comptonisation, so that its high-energy tail fits the soft X-ray excess. In recent years, multi-wavelength studies have found warm Comptonisation to be a viable explanation for the soft excess in Seyfert-1 AGN (e.g. most recently in Ark 120, Porquet et al. 2018). The fitted

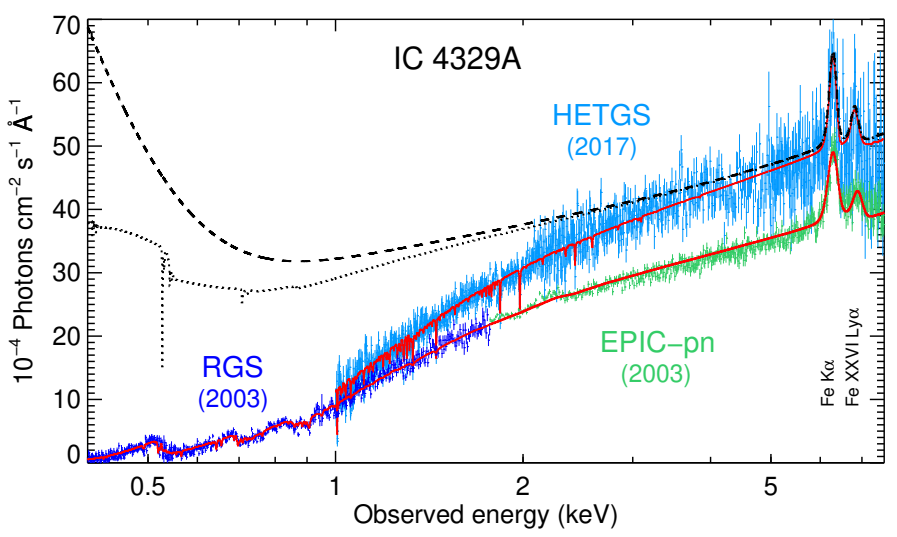

Fig. 2. Overview of the X-ray spectrum of IC 4329A taken with XMMNewton RGS and EPIC-pn in 2003, and Chandra HETGS in 2017. Our best-fit models to the data are also displayed (in red), with the powerlaw continuum being brighter in 2017. The underlying 2017 continuum model without any X-ray absorption is shown in dashed black line. For comparison, the continuum model with only absorption by the Milky Way is shown in dotted black line, which demonstrates the strong intrinsic absorption by IC 4329A.

parameters of the comt model are its normalisation, seed photons temperature $T_{\text {seed }}$, electron temperature $T_{\mathrm{e}}$, and optical depth $\tau$ of the up-scattering plasma. The primary continuum model components are shown in the SED of Fig. 3. The best-fit parameters of the power-law component, and the warm Comptonisation component (disk + soft X-ray excess), are provided in Table 2. The observed flux and intrinsic luminosity of IC 4329A over different energy bands are given in Table 3.

\subsection{Reprocessed X-ray emission}

The primary X-ray continuum undergoes reprocessing, which is evident by the presence of the $\mathrm{Fe} \mathrm{K} \alpha$ line in the HETGS and EPIC-pn spectra (Fig. 2, top panel). We measure a rest line energy of $6.39 \pm 0.01 \mathrm{keV}$ for this line, which is consistent with neutral $\mathrm{Fe}$ emission. The flux of the $\mathrm{Fe} \mathrm{K} \alpha$ line is $8 \pm 1 \times 10^{-13} \mathrm{erg} \mathrm{cm}^{-2} \mathrm{~s}^{-1}$. We applied an X-ray reflection component (refl), which reprocesses an incident power-law continuum to produce the $\mathrm{Fe} \mathrm{K} \alpha$ line and the Compton hump at hard X-rays. The refl model in SPEX computes the Fe K $\alpha$ line according to Zycki \& Czerny (1994), and the Compton-reflected continuum according to Magdziarz \& Zdziarski (1995), as described in Zycki et al. (1999). The photon index $\Gamma$ of the incident power-law was set to that of the observed primary continuum $(\Gamma=1.78)$. The exponential high-energy cut-off of this incident power-law is also set to that of the observed primary power-law component at $186 \mathrm{keV}$ (Brenneman et al. 2014). In our modelling the normalisation of the incident power-law continuum is set to the average of the HETGS and XMM-Newton observations. The ionisation parameter of refl is set to zero to produce a cold reflection component with all abundances kept at their solar values. The reflection scale $s$ parameter of the refl model was fitted. The refl component was convolved with a Gaussian velocity broadening model to fit the width $\sigma_{v}$ of the Fe $\mathrm{K} \alpha$ line, which is about $3400 \pm 500 \mathrm{~km} \mathrm{~s}^{-1}$. We do not require a relativistic line profile to fit the $\mathrm{Fe} \mathrm{K} \alpha$ line.

Apart from the $\mathrm{Fe} \mathrm{K} \alpha$ line, there is another emission line at rest energy $6.95 \pm 0.03 \mathrm{keV}$, which is consistent with a Fe XxvI Ly $\alpha$ line $(6.97 \mathrm{keV})$. We fitted this line with a simple Gaussian model. The flux of the Fe xxvi line is $3 \pm 1 \times 10^{-13} \mathrm{erg} \mathrm{cm}^{-2} \mathrm{~s}^{-1}$. The line profile is unresolved with $\sigma_{v}<2000 \mathrm{~km} \mathrm{~s}^{-1}$. 

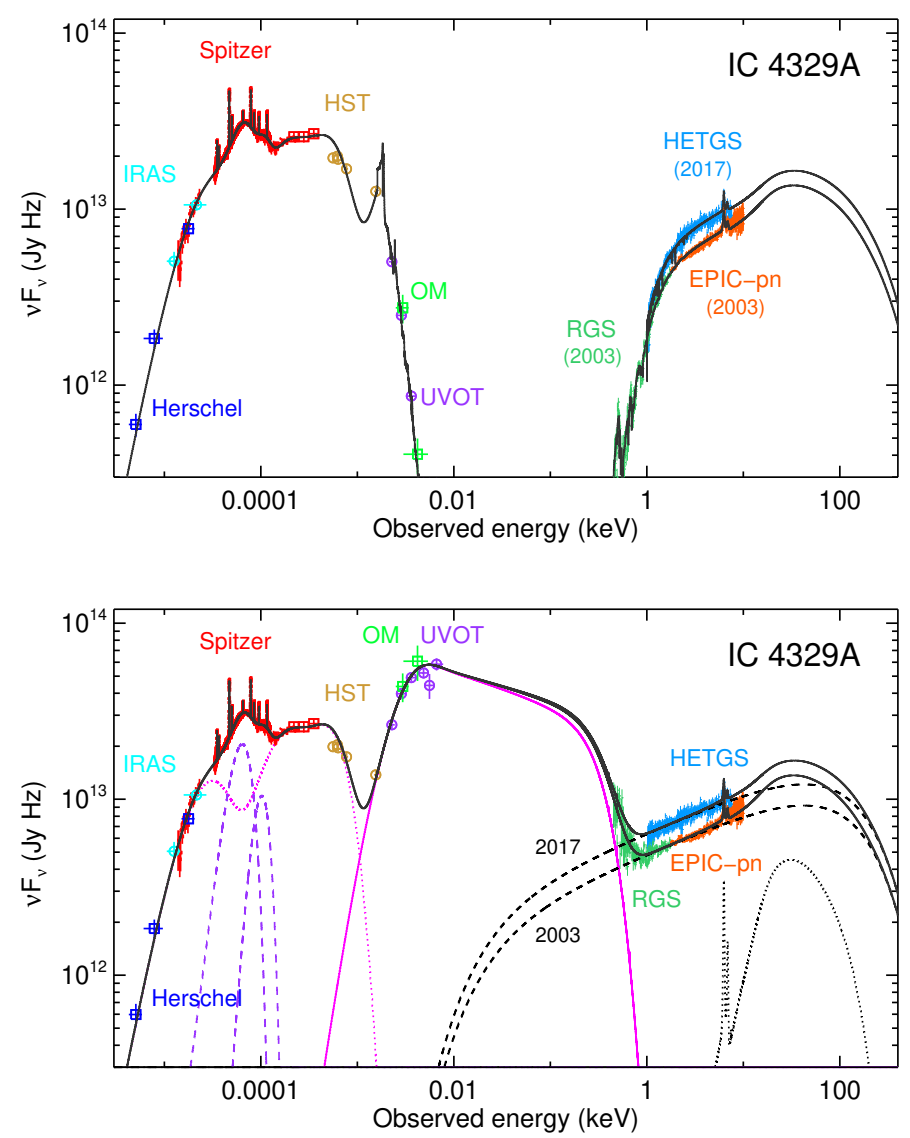

Fig. 3. SED of IC 4329A from far-IR to hard X-rays. The SED in the top panel includes the effects of reddening, X-ray absorption, host galaxy starlight emission, and the BLR and NLR emission. The SED in the bottom panel is corrected for these processes, revealing the underlying continuum. The best-fit model to the data is shown in both panels in solid black line. The contribution of individual continuum components are displayed in the bottom panel: a Comptonised disk component (solid magenta line), power-law continuum (dashed black line), X-ray reflection (dotted black line), thermal IR continuum (dotted magenta line). The model for the 9.7 and $18 \mu \mathrm{m}$ silicate features are also plotted (dashed purple lines). In the 2017 epoch (HETGS observation), the $\mathrm{X}$-ray power-law (dashed black line) is brighter than in the 2003 epoch (XMM-Newton observation).

\subsection{Thermal infrared continuum}

The primary AGN continuum drops from optical-UV towards lower energies. However, the observed continuum rises again from near-IR towards the mid-IR energies, and then declines again towards the far-IR energies (see Fig. 3). This is generally thought to be thermal emission from dust in AGN (e.g. Hernán-Caballero et al. 2016), which mainly comes from the AGN torus. To properly fit the broad-band continuum extending from near-IR to far-IR, we require three black body (bb) components, with different temperatures. The parameters of these components are given in Table 2. We note that we report on the 9.7 and $18 \mu \mathrm{m}$ dust features separately in Sect. 5.2.

\subsection{Emission from the $B L R$, the $N L R$, and the galactic bulge}

Apart from the optical and UV continuum, the photometric filters of OM and UVOT contain emission from the broadline region (BLR) and the narrow-line region (NLR) of the AGN. Therefore, in order to correct for this contamination, we applied the emission model derived in Mehdipour et al. (2015)
Table 2. Best-fit parameters of the broad-band continuum model components for IC 4329A.

\begin{tabular}{lc}
\hline \hline Parameter & Value \\
\hline \multicolumn{1}{c}{ Primary X-ray power-law component (pow): } \\
Normalisation & $1.77 \pm 0.01(2003)$ \\
& $2.33 \pm 0.01(2017)$ \\
Photon index $\Gamma$ & $1.78 \pm 0.01$ \\
\hline Disk component: optical-UV and the soft X-ray excess (comt): \\
Normalisation & $1.4 \pm 0.1$ \\
$T_{\text {seed }}(\mathrm{eV})$ & $0.8 \pm 0.1$ \\
$T_{\mathrm{e}}(\mathrm{eV})$ & $75 \pm 3$ \\
Optical depth $\tau$ & $33 \pm 2$ \\
\hline & $\mathrm{X}-\mathrm{ray}$ reflection $(\mathrm{refl}):$ \\
Incident power-law Norm. & $2.05(\mathrm{f})$ \\
Incident power-law $\Gamma$ & $1.78(\mathrm{f})$ \\
Reflection scale $s$ & $0.43 \pm 0.02$ \\
$\sigma_{v}\left(\mathrm{~km} \mathrm{~s} \mathrm{~s}^{-1}\right)$ & $3400 \pm 500$ \\
\hline \multicolumn{1}{c}{ Thermal IR emission components (bb): } \\
BB 1: $T(\mathrm{eV})$ & $0.0077 \pm 0.0001$ \\
BB 1: Flux & $1.59 \pm 0.02$ \\
BB 2: $T(\mathrm{eV})$ & $0.042 \pm 0.001$ \\
BB 2: Flux & $2.3 \pm 0.2$ \\
BB 3: $T(\mathrm{eV})$ & $0.121 \pm 0.003$ \\
BB 3: Flux & $3.4 \pm 0.4$ \\
&
\end{tabular}

Notes. The power-law normalisation of the pow and refl components is in units of $10^{52}$ photons $\mathrm{s}^{-1} \mathrm{keV}^{-1}$ at $1 \mathrm{keV}$. The normalisation of the Comptonised disk component (comt) is in units of $10^{57}$ photons $\mathrm{s}^{-1} \mathrm{keV}^{-1}$. The flux of the bb components are in $10^{-10} \mathrm{erg} \mathrm{cm}^{-2} \mathrm{~s}^{-1}$. The high-energy exponential cut-off of the power-law for both pow and refl is fixed to $186 \mathrm{keV}$. The photon index $\Gamma$ of the incident powerlaw for the reflection component (refl) is set to the $\Gamma$ of the observed primary power-law continuum (pow).

for NGC 5548 as a template model for the optical and UV data of IC 4329A. The model takes into account the Balmer continuum, the Fe II feature, and the emission lines from the BLR and NLR. We normalised this model to the intrinsic luminosity of the $\mathrm{H} \beta$ line in IC $4329 \mathrm{~A}$ as derived by Marziani et al. (1992), which is $2.4 \times 10^{42} \mathrm{erg} \mathrm{s}^{-1}$.

To take into account the host galaxy optical and UV stellar emission in the OM and UVOT filters, we used the galactic bulge model of Kinney et al. (1996), and normalised it to the IC 4329A host galaxy flux derived from HST images by Bentz et al. (2013). This is about $3.57 \times 10^{-15} \mathrm{erg} \mathrm{cm}^{-2} \mathrm{~s}^{-1} \AA^{-1}$ at $5100 \AA$.

Apart from the BLR and NLR emission in the optical and UV band, there is also infrared emission from the NLR, which is present in the Spitzer/IRS high-resolution spectrum (see Sect. 5.2). These narrow forbidden lines include [S Iv] $(10.511 \mu \mathrm{m}),[\mathrm{Ne}$ II] $(12.814 \mu \mathrm{m}),[\mathrm{Ne}$ v] $(14.322 \mu \mathrm{m}),[\mathrm{Ne}$ III] $(15.555 \mu \mathrm{m}),[\mathrm{S}$ III] $(18.713 \mu \mathrm{m}),[\mathrm{Ne} \mathrm{v}](24.318 \mu \mathrm{m})$, [O IV] $(25.913 \mu \mathrm{m})$. Therefore, in our SED modelling, we take into account these components by fitting narrow Gaussian emission lines to these lines.

\section{Modelling of the ISM and the AGN wind}

\subsection{X-ray absorption by the diffuse interstellar gas}

Prior to modelling the ISM gas absorption in IC 4329A, we first determined our model for the Milky Way absorption. The 
Table 3. Observed flux $(F)$ and intrinsic luminosity $(L)$ of IC 4329A over various energy bands.

\begin{tabular}{lcc}
\hline \hline Energy range & $\begin{array}{c}F \\
\left(10^{-11} \mathrm{erg} \mathrm{cm}^{-2} \mathrm{~s}^{-1}\right)\end{array}$ & $\begin{array}{c}L \\
\left(10^{44} \mathrm{erg} \mathrm{s}^{-1}\right)\end{array}$ \\
\hline Hard X-ray $(2-10 \mathrm{keV})$ & $11.0(2003)$ & $0.7(2003)$ \\
& $14.2(2017)$ & $0.9(2017)$ \\
Soft X-ray $(0.2-2 \mathrm{keV})$ & $3.0(2003)$ & $1.4(2003)$ \\
& $3.7(2017)$ & $1.6(2017)$ \\
EUV $(100-1000 \AA)$ & $8 \times 10^{-7}$ & 5.8 \\
UV $(1000-4000 \AA)$ & 0.2 & 4.5 \\
Optical $(4000-7000 \AA)$ & 2.2 & 1.0 \\
Near-IR $(0.7-3 \mu \mathrm{m})$ & 23.5 & 1.5 \\
Mid-IR $(3-40 \mu \mathrm{m})$ & 64.6 & 3.9 \\
Far-IR $(40-500 \mu \mathrm{m})$ & 10.8 & 0.7 \\
\hline 1-1000 Ryd & $16.9(2003)$ & $8.5(2003)$ \\
& $21.6(2017)$ & $9.1(2017)$ \\
Bolometric $\left(10^{-6}-10^{3} \mathrm{keV}\right)$ & $152.0(2003)$ & $24.5(2003)$ \\
& $164.9(2017)$ & $26.4(2017)$ \\
\hline
\end{tabular}

Notes. The values correspond to the SEDs shown in Fig. 3. The continuum modelling is described in Sect. 3 (Table 2). The observed fluxes $(F)$ include the effects of all the reddening and absorption components. The host galaxy optical and UV emission, and the AGN BLR and NLR emission, are excluded from the reported fluxes and luminosities. The IR fluxes and luminosities include all the dust emission components.

X-ray continuum and line absorption by the Galaxy are taken into account by applying the hot model in SPEX. This model calculates the transmission of a plasma in collisional ionisation equilibrium at a given temperature, which for neutral ISM is set to $0.5 \mathrm{eV}$. The Milky Way column density $N_{\mathrm{H}}$ is fixed to $4.61 \times 10^{20} \mathrm{~cm}^{-2}$ (Kalberla et al. 2005) in our line of sight to IC 4329A. The Galactic molecular $N_{\mathrm{H}}$ in our line of sight is a small fraction $(17 \%)$ of the total $N_{\mathrm{H}}$ according to Willingale et al. (2013).

Apart from the Milky Way absorption, an additional neutral ISM component intrinsic to the source is required for modelling the X-ray spectrum. This component is essential to fit the strong suppression of the soft X-ray continuum in IC 4329A (see Fig. 2). Also, such a component is needed to fit the absorption features of neutral gas in the spectrum (e.g. seen in O I). We thus incorporate another hot component with its temperature fixed to $0.5 \mathrm{eV}$ to fit the column density of neutral gas in IC $4329 \mathrm{~A}$, which is found to be $N_{\mathrm{H}}=3.1 \pm 0.2 \times 10^{21} \mathrm{~cm}^{-2}$. As this is a high column density of ISM in IC 4329A, a mildlyionised component is also detected through primarily $\mathrm{O}$ III. Thus, we include another hot component, with its temperature and $N_{\mathrm{H}}$ fitted. This mildly-ionised ISM component is found to have a temperature $T_{\mathrm{e}}=7.3 \pm 0.8 \mathrm{eV}$ with $N_{\mathrm{H}}=8 \pm 1 \times 10^{20} \mathrm{~cm}^{-2}$. In Sects. 4.2 and 5.3, we model additional components of the absorption in IC 4329A, produced by the AGN wind, and dust, respectively.

\subsection{X-ray absorption by the ionised AGN wind}

The RGS and HETGS spectra of IC 4329A exhibit a series of absorption lines belonging to outflowing ionised gas. We simultaneously modelled the RGS and HETGS spectra to derive a model for this ionised wind from the AGN. For photoionisation modelling and spectral fitting, we use the pion model in SPEX (see Mehdipour et al. 2016), which is a self-consistent model that calculates the thermal and ionisation balance together with the spectrum of a plasma in photoionisation equilibrium (PIE).
The pion model uses the SED (Sect. 3) from the continuum model components set in SPEX. During spectral fitting, as the continuum varies, the thermal and ionisation balance and the spectrum of the plasma are re-calculated at each stage. This means while using realistic broad-band continuum components to fit the data, the photoionisation is calculated accordingly by the pion model.

To properly fit all the absorption lines from various ionic species we require three photoionisation (pion) components with different values for the ionisation parameter $\xi$ (Tarter et al. 1969; Krolik et al. 1981). This parameter is defined as $\xi=L / n_{\mathrm{H}} r^{2}$, where $L$ is the luminosity of the ionising source over the $1-1000$ Ryd band $(13.6 \mathrm{eV}$ to $13.6 \mathrm{keV})$ in $\operatorname{erg~s}^{-1}, n_{\mathrm{H}}$ the hydrogen density in $\mathrm{cm}^{-3}$, and $r$ the distance between the photoionised gas and the ionising source in $\mathrm{cm}$. The pion components are added one at a time until all the observed features are modelled and thus our fit is no longer improved.

According to our model, the lowest ionisation component (Comp. A) produces absorption from the Li-like ion $\mathrm{O}$ VI, the He-like ions $\mathrm{C}$ v, $\mathrm{N}$ vi, and $\mathrm{O}$ vII, and the $\mathrm{H}$-like ion $\mathrm{C}$ vi. Moreover, this component is responsible for producing a shallow unresolved transition array (UTA, Behar et al. 2001) at about 16-17 $\AA$ from the M-shell Fe ions. The inclusion of this component improves our fit by $\Delta \mathrm{C}$-stat $=820$. The next ionisation component (Comp. B) primarily produces lines from the H-like $\mathrm{N}$ vII and $\mathrm{O}$ vIII, as well as the He-like $\mathrm{Ne}$ IX and $\mathrm{Mg}$ XI. The lines fitted by Comp. B provide a better fit by $\Delta \mathrm{C}$-stat $=440$. Finally, the highest ionisation component (Comp. C) produces the H-like ions Ne x, Mg xII, Si xIv, as well as the He-like Si xIII. This component also produces the high-ionisation $\mathrm{Fe}$ species in the form of Fe xIx, Fe xx, and FexxI. The addition of the high-ionisation component further improves the fit by $\Delta \mathrm{C}$-stat $=350$. The column density $N_{\mathrm{H}}$, the ionisation parameter $\xi$, the outflow velocity $v_{\text {out }}$, and the turbulent velocity $\sigma_{v}$ of each pion component are fitted. The covering fraction of all our absorption components in our modelling is fixed to unity. As the RGS and HETGS spectra are taken at different epochs, we allowed $N_{\mathrm{H}}$ and $\xi$ of the components to be different for the two epochs. The model transmission spectrum of the three pion components (Comps. A to C) are displayed in Fig. 4. The best-fit parameters of the AGN wind components, and the ISM components, are given in Table 4.

We fit all the X-ray absorption by the AGN wind in IC 4329A with three pion photoionisation components. However, in the X-ray analysis of IC 4329A by Steenbrugge et al. (2005), four photoionisation components were incorporated. The three highest ionisation components in their study roughly correspond to our three pion components, but they include an extra photoionisation component, with a very low ionisation $(\log \xi \sim-1.37)$ and zero outflow velocity, which we do not require in our modelling. This discrepancy between the two works can be explained by differences in the modelling of the neutral X-ray absorption in IC 4329A. The derived column density of the neutral ISM gas by Steenbrugge et al. (2005; $\left.N_{\mathrm{H}}=1.7 \times 10^{21} \mathrm{~cm}^{-2}\right)$ is significantly smaller than the one derived in our study $\left(N_{\mathrm{H}}=3.1 \times 10^{21} \mathrm{~cm}^{-2}\right)$. However, this difference in $N_{\mathrm{H}}$ is instead modelled by the cold photoionisation component in Steenbrugge et al. (2005), whereas we associate it to the ISM absorption in IC 4329A. Moreover, some differences in the parameterisation of the absorption components can be attributed to our inclusion of the dust model and the determination of the broad-band continuum, which result in a better fit to the spectra. Furthermore, there are extended updates and enhancements to the atomic database of SPEX v3 and the new 


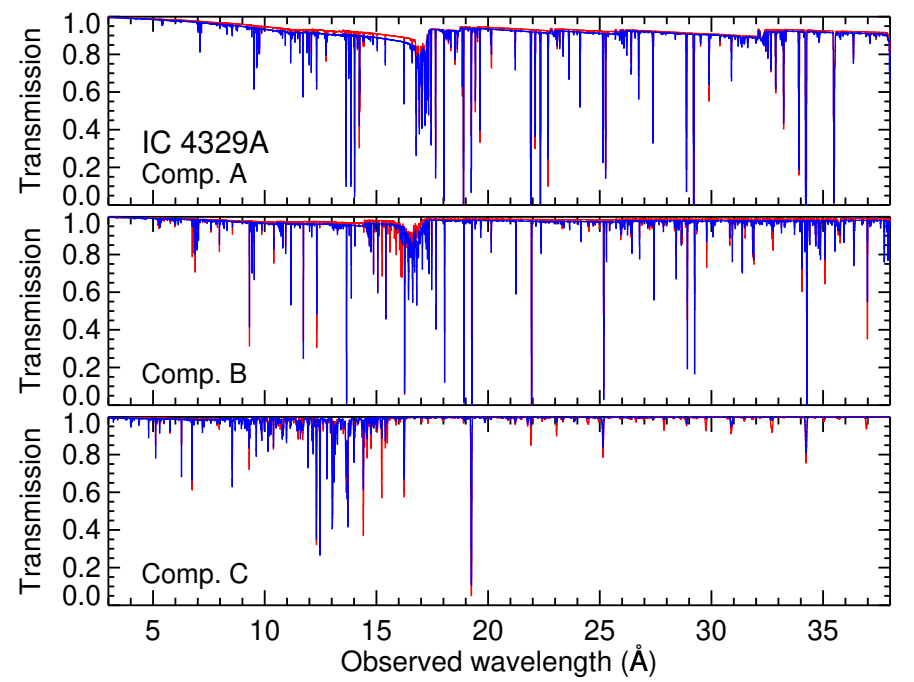

Fig. 4. Model transmission spectrum of the three components of the AGN wind in IC 4329A. Comp. A is the lowest-ionisation component, and Comp. $\mathrm{C}$ the highest. The model shown in blue is the one derived from the 2017 HETGS observations. The model derived from the archival 2003 XMM-Newton observations is also shown for comparison, which is plotted in red behind the blue one.

pion photoionisation model, which were not available back in SPEX v2, used by Steenbrugge et al. (2005).

\section{Multi-wavelength analysis of dust in IC 4329A}

\subsection{Dust reddening in IC $4329 \mathrm{~A}$}

IC 4329A displays significant internal reddening. This is evident from both the Balmer decrement (i.e. the observed $\mathrm{H} \alpha / \mathrm{H} \beta$ flux ratio) and the steepness of the optical-UV continuum (see Fig. 3, top panel). The Balmer decrement of IC 4329A has been reported in different papers to be from about 9 to 12 (Wilson \& Penston 1979; Marziani et al. 1992; Winge et al. 1996; Malkan et al. 2017). This is significantly higher than the expected Balmer decrement, implying significant internal reddening. The theoretical calculations for "Case B" recombination, where gas is optically thick in Lyman lines, give $\mathrm{H} \alpha / \mathrm{H} \beta \approx 2.85$ (Baker \& Menzel 1938; Osterbrock \& Ferland 2006). Moreover, the observations of unreddened AGN show that the Balmer decrement of the BLR in $\mathrm{AGN}$ is $\mathrm{H} \alpha / \mathrm{H} \beta \approx 2.72$ (Gaskell 2017). Importantly, the intrinsic reddening also affects the optical and UV continuum and changes the shape of the so-called "big-blue-bump" thermal emission from the accretion disk. Unlike SEDs of typical unreddened AGN (e.g. NGC 5548, Mehdipour et al. 2015), the flux of IC 4329A drops rapidly towards higher UV energies (Fig. 3, top panel), which is indicative of strong reddening.

Before deriving the reddening in IC 4329A, we first fixed our model for reddening in the ISM of the Milky Way, which in our line of sight has a colour excess $E(B-V)=0.052 \mathrm{mag}$ (Schlafly \& Finkbeiner 2011). We applied the ebv model in SPEX to model this reddening, which incorporates the extinction curve of Cardelli et al. (1989), including the update for near-UV given by O'Donnell (1994). The scalar specifying the ratio of total to selective extinction $R_{\mathrm{V}}=A_{\mathrm{V}} / E(B-V)$ was fixed to 3.1.

A general model for internal reddening in AGN is lacking. Studies of reddening in AGN have found different kinds of extinction laws. For example, Hopkins et al. (2004) examined the SEDs of a large sample of quasars using broad-band
Table 4. Best-fit parameters of the final model for the AGN wind and the ISM in IC 4329A.

\begin{tabular}{|c|c|}
\hline Parameter & Value \\
\hline \multicolumn{2}{|c|}{ AGN wind Comp. A (pion): } \\
\hline \multirow{2}{*}{$N_{\mathrm{H}}\left(10^{20} \mathrm{~cm}^{-2}\right)$} & $7 \pm 1(2003)$ \\
\hline & $8 \pm 1(2017)$ \\
\hline \multirow[t]{2}{*}{$\log \xi\left(\mathrm{erg} \mathrm{cm} \mathrm{s}^{-1}\right)$} & $0.8 \pm 0.1(2003)$ \\
\hline & $1.0 \pm 0.1(2017)$ \\
\hline$v_{\text {out }}\left(\mathrm{km} \mathrm{s}^{-1}\right)$ & $-410 \pm 30$ \\
\hline$\sigma_{v}\left(\mathrm{~km} \mathrm{~s}^{-1}\right)$ & $90 \pm 20$ \\
\hline \multicolumn{2}{|c|}{ AGN wind Comp. B (pion): } \\
\hline \multirow[t]{2}{*}{$N_{\mathrm{H}}\left(10^{20} \mathrm{~cm}^{-2}\right)$} & $6 \pm 1(2003)$ \\
\hline & $6 \pm 1(2017)$ \\
\hline \multirow[t]{2}{*}{$\log \xi\left(\mathrm{erg} \mathrm{cm} \mathrm{s}^{-1}\right)$} & $2.1 \pm 0.1(2003)$ \\
\hline & $1.9 \pm 0.1(2017)$ \\
\hline$v_{\text {out }}\left(\mathrm{km} \mathrm{s}^{-1}\right)$ & $0 \pm 20$ \\
\hline$\sigma_{v}\left(\mathrm{~km} \mathrm{~s}^{-1}\right)$ & $60 \pm 10$ \\
\hline \multicolumn{2}{|c|}{ AGN wind Comp. C (pion): } \\
\hline \multirow{2}{*}{$N_{\mathrm{H}}\left(10^{20} \mathrm{~cm}^{-2}\right)$} & $11 \pm 2(2003)$ \\
\hline & $22 \pm 2(2017)$ \\
\hline \multirow[t]{2}{*}{$\log \xi\left(\mathrm{erg} \mathrm{cm} \mathrm{s}^{-1}\right)$} & $2.79 \pm 0.07(2003)$ \\
\hline & $3.03 \pm 0.03(2017)$ \\
\hline$v_{\text {out }}\left(\mathrm{km} \mathrm{s}^{-1}\right)$ & $-360 \pm 20$ \\
\hline$\sigma_{v}\left(\mathrm{~km} \mathrm{~s}^{-1}\right)$ & $210 \pm 40$ \\
\hline \multicolumn{2}{|c|}{ Neutral ISM component of IC 4329A (hot): } \\
\hline$N_{\mathrm{H}}\left(10^{20} \mathrm{~cm}^{-2}\right)$ & $31 \pm 1$ \\
\hline$T_{\mathrm{e}}(\mathrm{eV})$ & $0.5(\mathrm{f})$ \\
\hline \multicolumn{2}{|c|}{ Mildly-ionised ISM component of IC 4329A (hot): } \\
\hline$N_{\mathrm{H}}\left(10^{20} \mathrm{~cm}^{-2}\right)$ & $8 \pm 1$ \\
\hline$T_{\mathrm{e}}(\mathrm{eV})$ & $7.3 \pm 0.8$ \\
\hline \multicolumn{2}{|c|}{ C-stat/d.o.f. $=10069 / 9280$} \\
\hline
\end{tabular}

Notes. The parameters of the associated dust model in IC 4329A are presented in Table 5.

photometry data from SDSS. They concluded that the reddening is best described by SMC-like extinction. On the other hand, the Czerny et al. (2004) study of composite quasar spectra from SDSS finds a "grey" (flat) extinction curve, where the extinction curve is flatter than that of the diffuse Milky Way. They also find no trace of the " $2175 \AA$ bump" that is seen in the Milky Way extinction curve. On the other hand, from a case study of a reddened AGN (NGC 3227) using HST, and comparing its spectrum with that of an unreddened AGN (NGC 4151), Crenshaw et al. (2001) concluded that the extinction curve in the UV is even steeper than that of SMC. On the other hand, flat extinction curves have been found by Maiolino et al. (2001), Gaskell et al. (2004) and Gaskell \& Benker (2007).

We determined the colour excess $E(B-V)$ in IC 4329A by jointly modelling the reddening of the optical-UV continuum and the Balmer decrement. We tested different template extinction laws from Cardelli et al. (1989; Milky Way), Czerny et al. (2004; flat), and Zafar et al. (2015; steep). The user-defined multiplicative model in SPEX (musr) was used to import these reddening models into SPEX. We find that a flat extinction curve (Czerny et al. 2004) with $E(B-V)=1.0 \pm 0.1$ is most appropriate for IC 4329A. It gives the best-fit to the broad-band continuum with the most reasonable continuum parameters. The steeper extinction curves lead to unphysical UVEUV continuum luminosity. This conclusion was also reached for the reddened AGN ESO 113-G010 (Mehdipour et al. 2012). 


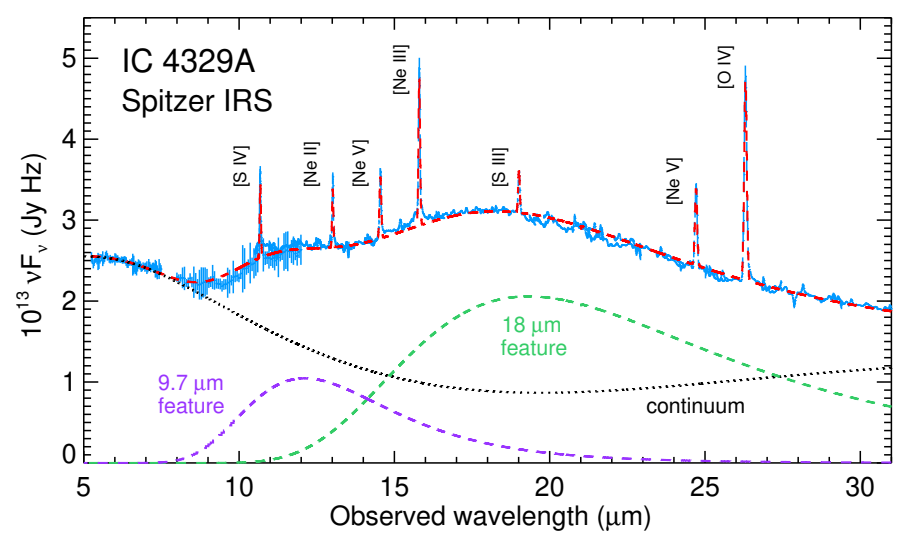

Fig. 5. Spitzer IRS spectrum of IC 4329A. The 9.7- and 18-micron emission features are shown in dashed purple and green lines, respectively. The best-fit model to the data, which includes the narrow emission lines from the AGN NLR, is shown in dashed red line. The underlying thermal IR continuum model is shown in dotted black line for comparison.

Therefore, in our modelling of IC 4329A, we adopt the extinction curve of Czerny et al. (2004), with $R_{\mathrm{V}}$ fixed to 3.1. In this case the relation between reddening and the Balmer decrement is $E(B-V)=1.475 \log \left(R_{\text {obs }} / R_{\text {int }}\right)$, where $R_{\text {obs }}$ and $R_{\text {int }}$ are the observed and intrinsic $\mathrm{H} \alpha / \mathrm{H} \beta$, respectively. Thus, using the reported range of the Balmer decrement in IC 4329A $\left(R_{\text {obs }} \approx 9\right.$ to 12$)$, and $R_{\text {int }}=2.72$, the reddening $E(B-V)$ ranges between about 0.8 and 1.0. This matches $E(B-V)=1.0 \pm 0.1$ derived from de-reddening of the continuum (see Fig. 3). We later discuss the origin of the flat extinction curve in Sect. 6.

\subsection{Dust IR emission features in IC $4329 A$}

The widely known 9.7 and $18 \mu \mathrm{m}$ features in the mid-IR spectra of AGN belong to silicate dust (e.g. Sturm et al. 2005; Henning 2010). The $9.7 \mu \mathrm{m}$ feature is generally attributed to the stretching of the $\mathrm{Si}-\mathrm{O}$ bonds in silicates, while the $18 \mu \mathrm{m}$ feature is attributed to $\mathrm{O}-\mathrm{Si}-\mathrm{O}$ bending in the same material (e.g. Henning 2010). These features have canonical wavelengths of 9.7 and $18 \mu \mathrm{m}$ in the Milky Way diffuse ISM, however, in AGN they often show deviation to longer wavelengths (e.g. Sturm et al. 2005; Hatziminaoglou et al. 2015). They also display much broader spectral width compared to that of the Milky Way (Li et al. 2008). These features are thought to originate from silicates in the AGN dusty torus (e.g. Pier \& Krolik 1992, 1993; Siebenmorgen et al. 2005; Mason 2015).

The 9.7 and $18 \mu \mathrm{m}$ silicate features are evident in emission in the Spitzer IRS spectrum of IC 4329A (Fig. 5). The presence of these emission features in IC 4329A were also previously reported by the AGN sample studies with Spitzer (e.g. Gallimore et al. 2010). In our broad-band SED modelling, we fit these features by the means of Gaussian emission components (gaus). This parametrisation allows us to determine the peak wavelength, flux, and the spectral width of these features. Our best-fit model to the Spitzer IRS spectrum is shown in Fig. 5. The results of our modelling are given in Table 5, and are discussed in Sect. 6.3.

\subsection{Dust X-ray absorption features in IC $4329 A$}

A telltale sign of dust X-ray absorption is that despite atomic and ionic absorption, the photoelectric edges in the X-ray spectrum are not well fitted. This is because dust modifies the profile
Table 5. Best-fit parameters of the dust model in IC 4329A.

\begin{tabular}{|c|c|}
\hline Parameter & Value \\
\hline \multicolumn{2}{|c|}{ Reddening component of IC 4329A (musr): } \\
\hline$E(B-V)$ & $1.0 \pm 0.1$ \\
\hline \multicolumn{2}{|c|}{$\begin{array}{l}\text { Dust IR emission features in IC 4329A (gaus) } \\
\qquad 9.7 \mu \mathrm{m} \text { feature: }\end{array}$} \\
\hline$\lambda_{\text {peak }}(\mu \mathrm{m})$ & $13 \pm 1$ \\
\hline FWHM $(\mu \mathrm{m})$ & $7 \pm 3$ \\
\hline Flux $\left(10^{-11} \mathrm{erg} \mathrm{cm}^{-2} \mathrm{~s}^{-1}\right)$ & $5 \pm 1$ \\
\hline \multicolumn{2}{|c|}{$18 \mu \mathrm{m}$ feature: } \\
\hline$\lambda_{\text {peak }}(\mu \mathrm{m})$ & $22 \pm 1$ \\
\hline FWHM $(\mu \mathrm{m})$ & $18 \pm 1$ \\
\hline Flux $\left(10^{-11} \mathrm{erg} \mathrm{cm}^{-2} \mathrm{~s}^{-1}\right)$ & $14 \pm 1$ \\
\hline \multicolumn{2}{|c|}{ Dust X-ray absorption in IC 4329A (amol): } \\
\hline$N_{\text {O-dust }}\left(10^{17} \mathrm{~cm}^{-2}\right)$ & $6.2 \pm 0.6$ \\
\hline$N_{\text {Si-dust }}\left(10^{17} \mathrm{~cm}^{-2}\right)$ & $1.9 \pm 0.4$ \\
\hline$N_{\mathrm{Fe}-\text { dust }}\left(10^{17} \mathrm{~cm}^{-2}\right)$ & $1.5 \pm 0.1$ \\
\hline
\end{tabular}

Notes. The AGN extinction curve of Czerny et al. (2004) is used to derive the reddening $E(B-V)$ with $R_{V}$ fixed at 3.1. The $\lambda_{\text {peak }}$ wavelengths correspond to the rest-frame of IC 4329A.

of the absorption edges. In the case of IC 4329A, despite neutral and warm absorption by the ISM of the host galaxy, and the ionised gas absorption by the AGN wind, additional absorption is required at the K edge of $\mathrm{O}$, the LII and LIII edges of Fe, and to lesser extent at the K edge of Si (see Fig. 6). Such stronger than expected edges are indicative of absorption by dust. The wavelength, absorption profile, and strength of these features are not consistent with atomic or ionic gas. Furthermore, the Milky Way column density and reddening in our line of sight to IC 4329A is too low to be responsible for these features. Therefore, they can only be attributed to dust grains in our line of sight in IC 4329A, which contain $\mathrm{O}, \mathrm{Si}$, and $\mathrm{Fe}$.

We use the amol model in SPEX to calculate the X-ray absorption by dust (see Pinto et al. 2010; Costantini et al. 2012). In our modelling, we deplete the $\mathrm{O}, \mathrm{Si}$, and $\mathrm{Fe}$ elements from gas to dust form. For absorption in IC 4329A the depletion factors are fitted, whereas for that of the Milky Way they are fixed. For the Milky Way contribution (albeit relatively tiny), the depletion factors are fixed to standard values taken from Jenkins (2009): $22 \%$ for $\mathrm{O}, 68 \%$ for $\mathrm{Si}$, and $94 \%$ for $\mathrm{Fe}$. We apply a simple template model (hematite $\mathrm{Fe}_{2} \mathrm{O}_{3}$ ) to incorporate dust absorption by $\mathrm{O}$ and $\mathrm{Fe}$ by fitting the $\mathrm{K}$ edge of $\mathrm{O}$ and the LII and LIII edges of $\mathrm{Fe}$. The inclusion of this dust model improves the fit to the edges significantly with $\Delta \mathrm{C}$-stat $=292$. While the Fe edges were fitted well at this stage, there were some remaining residuals at the $\mathrm{O}$ edge, which we found to be fitted well with the inclusion of molecular oxygen. This improved the fit further by $\Delta \mathrm{C}$-stat $=133$. Finally, we find that the fit to the Si K-edge is slightly improved by $\Delta \mathrm{C}$-stat $=30$ with the inclusion of crystal $\mathrm{Si}$. We note that using AGN spectra from the existing high-resolution $\mathrm{X}$-ray spectrometers, we can only constrain the column density of these elements in dust form, rather than determining the exact chemical composition of dust. Therefore, the above model represents an ad-hoc template model. Visual comparison of the bestfit models to the spectra with and without the dust model are shown in Fig. 6. The best-fit model with dust absorption (red line) fits the $\mathrm{O} \mathrm{K}$ and Fe LIII edges significantly better than the one without dust (black line). The AGN wind parameters are not significantly affected by the inclusion of dust as their absorption lines are similarly fitted well regardless of the dust model. The 

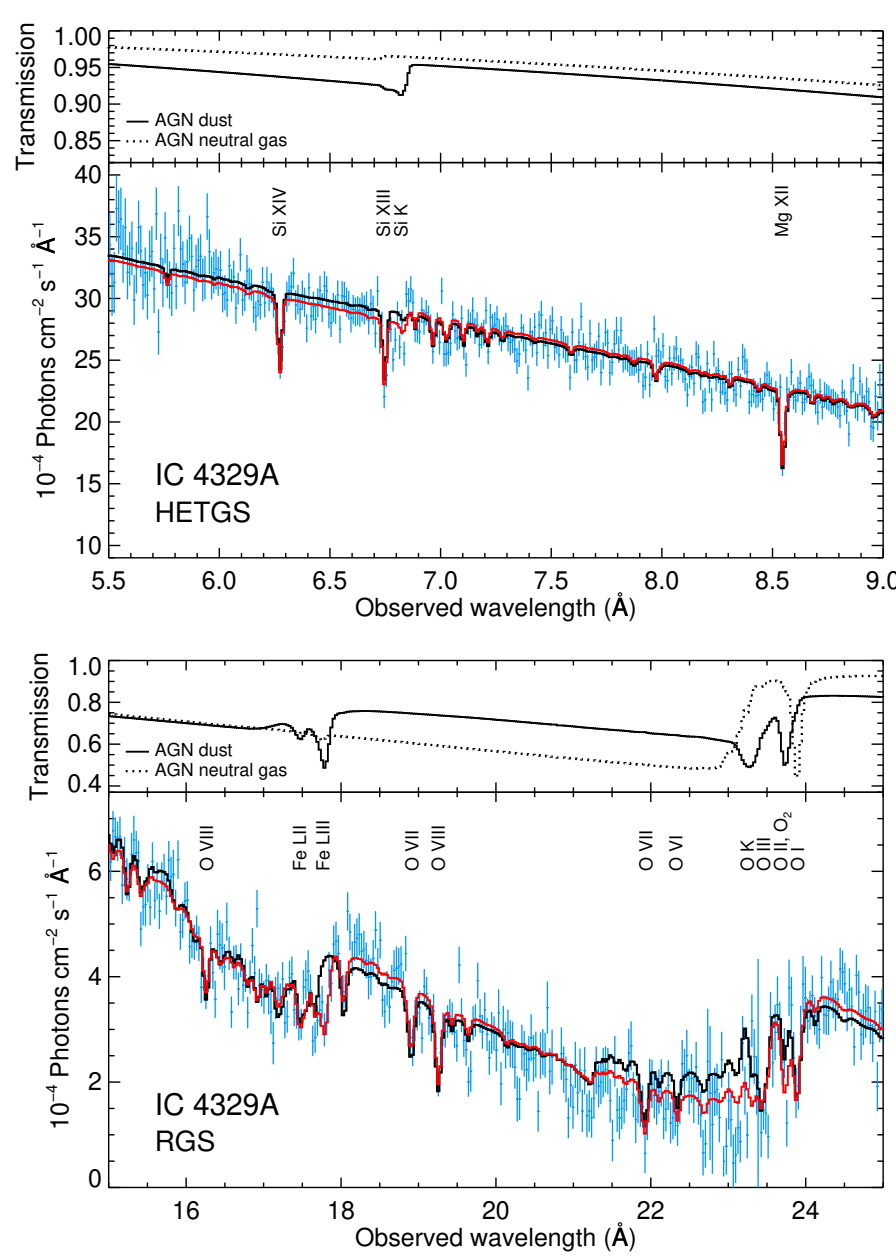

Fig. 6. Stacked HETGS (top panel) and RGS (bottom panel) spectra of IC 4329A in the $K$-band of Si (HETGS) and O (RGS), and the $L$-band of Fe (RGS). The best-fit model shown in red includes dust, whereas the one in black does not include dust. The panels above each spectrum show the transmission model by the $\mathrm{Si}, \mathrm{O}$, and $\mathrm{Fe}$ elements in atomic (dotted line) and dust (solid line) form in IC 4329A. The strongest absorption features in the spectrum are labelled, including the K-edge of $\mathrm{O}$ and $\mathrm{Si}$, and the LII- and LIII-edges of Fe from dust in the AGN, as well as lines from the ionised AGN wind. The model with dust absorption (shown in red) fits the $\mathrm{O} \mathrm{K}$ and Fe LIII edges significantly better than the one without dust (shown in black).

obtained best-fit column densities of elements in dust form in IC 4329A are given in Table 5. We discuss the results in Sect. 6.2.

\section{Discussion}

\subsection{Broad-band continuum of IC $4329 A$}

The broad-band continuum of IC 4329A is strongly modified by internal reddening and X-ray absorption. By modelling these effects in this paper, the underlying intrinsic emission of the AGN is uncovered. While the observed SED of IC 4329A appears significantly different from that of the archetypal Seyfert-1 galaxy NGC 5548, the underlying continuum from near-IR to hard X-rays is consistent with the global model derived for NGC 5548 in Mehdipour et al. (2015). The continuum from near-IR to soft X-rays can be explained by a single component, which Compton up-scatters the disk photons in an optically-thick, warm corona. The high-energy tail of this component produces the soft X-ray excess. The hard X-ray spectrum of IC 4329A is consistent with a typical power-law $(\Gamma \approx 1.78)$, produced in an optically-thin, hot corona, which is accompanied by a neutral X-ray reflection component. The accretion-powered radiation is reprocessed into lower energies by the dusty AGN torus, which its luminosity peaks at the midIR band. The broad-band modelling done in this paper enables us to derive the bolometric luminosity of the AGN, which is about $2.45 \times 10^{45} \mathrm{erg} \mathrm{s}^{-1}$ in 2003 , and $2.64 \times 10^{45} \mathrm{erg} \mathrm{s}^{-1}$ in 2017 (Table 3). Taking into account the black hole mass of IC 4329A $\left(1-2 \times 10^{8} M_{\odot}\right)$, these corresponds to bolometric luminosities at about $10-20 \%$ of the Eddington luminosity. The reprocessed emission by the AGN torus accounts for about $20 \%$ of the bolometric luminosity.

\subsection{Components of dust in IC $4329 A$}

In this paper we have carried out broad-band continuum modelling, together with X-ray and IR spectroscopy, to study dust in IC 4329A. By examining our results from reddening, X-ray absorption and IR emission by dust, and comparing with previous polarisation studies, we can construct a physical picture of dust in IC 4329A. We argue that the derived information from each of the above analyses points to the presence of two distinct components of dust in IC 4329A: one in the ISM of the host galaxy and its associated dust lane, and the other a nuclear component in the AGN torus with likely association to the wind. In Fig. 7 we illustrate how our line of sight towards the central engine is intercepted by these dust regions in IC 4329A.

The relationship between the hydrogen column density $N_{\mathrm{H}}$ and the reddening $E(B-V)$ can be written as $N_{\mathrm{H}}\left(\mathrm{cm}^{-2}\right)=a \times 10^{21} E(B-V)(\mathrm{mag})$, where $a$ is reported to have a value ranging from about 5.5 to 6.9 (Bohlin et al. 1978; Gorenstein 1975; Predehl \& Schmitt 1995; Güver \& Özel 2009). Therefore, for our derived $E(B-V)=1.0 \pm 0.1$, the expected $N_{\mathrm{H}}$ ranges between 5.0 and $7.6 \times 10^{21} \mathrm{~cm}^{-2}$. However, according to our modelling of the X-ray spectrum, the column density of neutral gas in IC 4329A is $N_{\mathrm{H}}=3.1 \pm 0.1 \times 10^{21} \mathrm{~cm}^{-2}$. Thus, in IC 4329A the neutral gas alone is not sufficient to produce all the observed reddening. On the other hand, if one considers that part of the dust that causes the reddening is associated to the ionised gas in IC 4329A (Table 4), in particular the less-ionised components of the wind (i.e. a dusty warm absorber), the sum of $N_{\mathrm{H}}$ matches the expected $N_{\mathrm{H}}$ inferred from the observed reddening. The AGN wind Comps. A and B, and the neutral+warm ISM give a total $N_{\mathrm{H}} 5.2 \pm 0.2 \times 10^{21} \mathrm{~cm}^{-2}$ (from the $2003 \mathrm{XMM}$ Newton spectra) and $N_{\mathrm{H}} 5.3 \pm 0.2 \times 10^{21} \mathrm{~cm}^{-2}$ (from the 2017 HETGS spectra). This excludes the highest ionisation component of the AGN wind. Therefore, the above results suggest that apart from the neutral ISM, the ionised gas (i.e. the warm ISM and the AGN wind) are likely dusty.

Apart from the above $N_{\mathrm{H}}$-reddening relation, the measured column densities of dust (Table 5) also indicate that some of the dust is likely associated to the AGN wind. This is mainly because the column density of Fe in dust form is too high to be feasible with Fe depletion in the ISM alone (for both the neutral and mildly-ionised components). In other words, the measured number of $\mathrm{Fe}$ atoms in dust form exceeds the number of $\mathrm{Fe}$ atoms available in gas form in the ISM. However, by considering the total $N_{\mathrm{H}}$, including that of the AGN wind, feasible depletion factors are obtained. Taking into account the possible range of the total $N_{\mathrm{H}}\left(4.8\right.$ to $\left.5.7 \times 10^{21} \mathrm{~cm}^{-2}\right)$, and the uncertainties on the column densities of O-dust, Si-dust, and Fe-dust, we derive the following depletion factors from gas to dust form in IC 4329A: $17-23 \%$ for O, 70-100\% for $\mathrm{Si}$, and $77-98 \%$ for $\mathrm{Fe}$. 


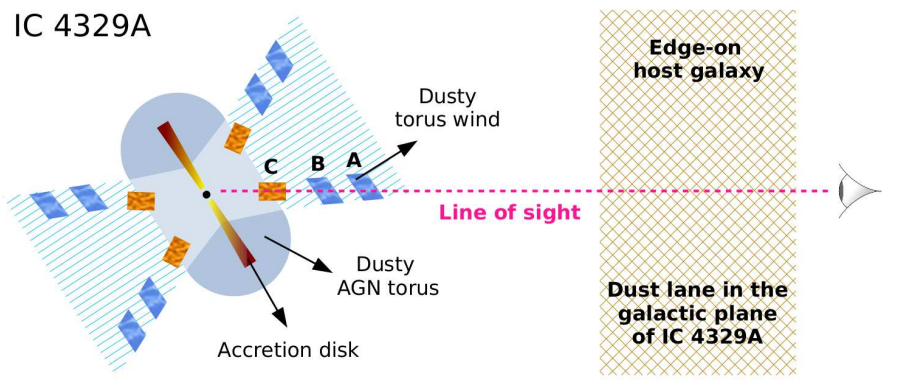

Fig. 7. Illustration of our line of sight through IC 4329A. The host galaxy and its dust lane are viewed edge-on, whereas the AGN disk and torus are tilted towards us. Our line of sight goes through the nuclear wind from the torus. The Comps. A and B of the AGN wind are located outside of the torus and are likely dusty.

These depletion factors are for the total gas in IC 4329A, and assume the proto-solar abundances of Lodders et al. (2009). The depletion factors are comparable to those in the diffuse Milky Way (e.g. Jenkins 2009). We further discuss the possibility of a dusty warm absorber from the AGN torus in Sect. 6.4.

\subsection{Dusty AGN torus}

IC 4329A displays significant optical polarisation (Martin et al. 1982; Wolstencroft et al. 1995). From imaging polarimetry of IC 4329A, Wolstencroft et al. (1995) find two components of polarisation. The first component is parallel to the galactic plane and the edge-on dust lane. The second component is a nuclear polarising component with a position angle approximately parallel to the galactic plane. The authors suggest that the first component arises from magnetic field in the plane of the galaxy, while the second component arises from dust scattering by an asymmetric geometry in the nucleus involving the AGN torus. Based on the polarisation of the nuclear component, Wolstencroft et al. (1995) argue that the torus must be significantly tilted in our line of sight, while we can still see the central engine. In this case, IC 4329A is still perceived spectroscopically as a type-1 AGN, but the inner edge of the torus will be viewed as an elliptical ring which introduces an asymmetry into the scattering geometry.

The 9.7 and $18 \mu \mathrm{m}$ silicate features are commonly seen in absorption in type-2 AGN, and in emission in type-1 AGN. This is because of strong silicate absorption by the AGN torus in type2 AGN, which obscures the line of sight. However, in IC 4329A, our view of the accretion disk is only partially obscured by the torus. Thus, silicate emission from the inner walls of the torus dominates over any weaker silicate absorption in our line of sight. Furthermore, highly-inclined (i.e. edge-on) galaxies often show the 9.7 and $18 \mu \mathrm{m}$ features in absorption, rather than emission (e.g. Alonso-Herrero et al. 2011). This can be attributed to silicate absorption in the disk of the galaxy. However, in IC $4329 \mathrm{~A}$ the 9.7 and $18 \mu \mathrm{m}$ features are seen in emission, rather than absorption (Deo et al. 2009; Alonso-Herrero et al. 2011), which is also found by our analysis of the Spitzer/IRS spectrum (Fig. 5). As suggested by Deo et al. (2009), this is likely because our line of sight does not intersect dense dust clouds in the ISM of the galaxy.

Tristram et al. (2009) have carried out a survey with MIDinfrared Interferometric instrument (MIDI) at the VLTI on 13 bright AGN (including IC 4329A). This is to resolve the nuclear dust emission in these AGN and study their spatial distribution. However, they find that IC 4329A emission is unresolved, which implies that the bulk of the mid-IR emission $(12 \mu \mathrm{m})$ is concentrated at $<10.8 \mathrm{pc}$ (Tristram et al. 2009). This suggests that mid-IR dust emission from the nucleus (i.e. the dusty torus) dominates over non-nuclear emission (i.e. the dust lane).

From our modelling of internal reddening in IC 4329A (Sect. 5.1), we derived $E(B-V) \approx 1.0$. The reddening is best described with a grey extinction curve (Czerny et al. 2004), which is relatively flat in the UV with no steep rise into the farUV. This implies that dust in IC 4329A is different from that of the Milky Way. From the Spitzer study of dust features in a sample of AGN, Xie et al. (2017) conclude that all sources require that the dust grains are micron-sized (typically $\sim 1.5$ micron), which is much larger than the sub-micron sized Galactic interstellar grains. This would imply a grey (flat) extinction law for AGN (Xie et al. 2017), which is what we find for IC 4329A from our SED modelling. Therefore, the torus dust grains in IC 4329A are likely larger than those in the diffuse Milky Way.

In IC 4329A, we find that the 9.7 and $18 \mu \mathrm{m}$ silicate emission features peak at wavelengths higher than the standard ISM silicate dust (Table 5). A recent census of the silicate features in the mid-IR spectra of AGN by Hatziminaoglou et al. (2015) shows that $\lambda_{\text {peak }}$ of the $9.7 \mu \mathrm{m}$ emission feature (in the rest frame) is often shifted to longer wavelengths relative to the nominal wavelength of $9.7 \mu \mathrm{m}$. This shift is much less present when the feature is in absorption. Hatziminaoglou et al. (2015) report that the shift nearly always occurs in AGN dominated spectra, where the fractional contribution of the AGN component to the total luminosity between 5 and $15 \mu \mathrm{m}$ is $>0.7$. From our modelling we find this is indeed the case for IC 4329A, as this fraction is $\approx 0.80$. The peak wavelength, spectral width, and relative strength of the 9.7 and $18 \mu \mathrm{m}$ features are thought to depend on the grain composition and size (e.g. Xie et al. 2017). For example, amorphous olivine gives the longest peak wavelength for the $9.7 \mu \mathrm{m}$ feature and the highest ratio of the $18 \mu \mathrm{m}$ feature to the $9.7 \mu \mathrm{m}$ feature (Xie et al. 2017), which is the case for IC 4329A. Moreover, the shifting and broadening of the silicate features have been explained by Li et al. (2008) using porous composite dust, consisting of amorphous silicate. They suggest that such porous dust is expected in the dense circumnuclear region of the AGN, as a consequence of grain coagulation.

\subsection{Dusty wind from the AGN torus}

From our photoionisation modelling of the AGN wind in IC 4329A (Sect. 4), we find that it consists of three ionisation components. According to our modelling, the two lowest ionisation components (Comps. A and B) are consistent with having no significant variability between the 2003 and 2017 epochs. Their column density $N_{\mathrm{H}}$ and ionisation parameter $\xi$ remain unchanged (Table 4). However, the highest ionisation component (Comp. C) displays significant variability in $N_{\mathrm{H}}$ and $\xi$. This increase in $\xi$ of Comp. C between the two epochs matches the increase in the luminosity of the X-ray continuum (Fig. 3, Tables 2 and 3), suggesting that Comp. $C$ has responded to the variability of the ionising SED. Therefore, the recombination timescale $\left(t_{\text {rec }}\right)$ of Comp. C needs to be shorter than the spacing between the two epochs, while the lack of variability in Comps. $\mathrm{A}$ and B suggests that their $t_{\mathrm{rec}}$ are longer than this limit. From our photoionisation modelling, the product $t_{\mathrm{rec}} \times n_{\mathrm{H}}$ for each component is yielded. We find $t_{\mathrm{rec}}$ is about $60 n_{4}^{-1}$ days for Comp. A, $49 n_{4}^{-1}$ days for Comp. B, and $9 n_{4}^{-1}$ days for Comp. C, where $n_{4}$ represents $n_{\mathrm{H}}=10^{4} \mathrm{~cm}^{-3}$. Hence, from the limit on $t_{\mathrm{rec}}$, based on the non-variability of Comps. A and B, and variability of Comp. $\mathrm{C}$, we can place a limit on the density $n_{\mathrm{H}}$ of each component. 
Consequently, from this $n_{\mathrm{H}}$ limit and the definition of the ionisation parameter $\left(\xi=L / n_{\mathrm{H}} r^{2}\right)$, limit on the location $r$ of each component from the ionising source is computed. We find Comp. A is at $r>350 \mathrm{pc}$, Comp. B at $r>83 \mathrm{pc}$, and Comp. C at $r<93 \mathrm{pc}$. For comparison, the inner radius of the AGN torus in IC 4329A, derived by the torus modelling of Alonso-Herrero et al. (2011) is $2.7 \mathrm{pc}$.

The observational characteristics of the wind in IC 4329A match the warm-absorber type winds in type-1 AGN. Such winds are most consistent with an origin from the AGN torus (e.g. Kaastra et al. 2012; Mehdipour et al. 2018). The location of the wind components in IC 4329A (Comps. A and B), and their relatively low outflow velocities, are consistent with a torus wind. As discussed in Sect. 6.2, a component of dust in IC 4329A arises from the AGN torus. Thus, the reddening and $\mathrm{X}$-ray absorption from this nuclear component in our line of sight is most likely associated to a wind from the torus. The two lowest ionisation components (Comps. A and B) of the AGN wind are most likely dusty. These components, which are located outside of the dusty torus, are beyond the dust sublimation radius, and therefore they can host dust. Interestingly, there are observational evidence of extended mid-IR emission from dust along the polar directions in type-2 AGN, such as the Circinus galaxy (Tristram et al. 2014), which may be produced by the dusty outflows from the AGN torus.

Currently using XMM-Newton and Chandra, the dust $\mathrm{X}$-ray features are only detectable in a very few X-ray bright AGN. The proposed Arcus mission (Smith et al. 2016) with its unprecedented sensitivity and energy resolution in the soft $\mathrm{X}$-ray band would provide a major breakthrough in the X-ray spectroscopy of dust. It would enable us to distinguish between different chemical compositions of dust in AGN, providing important insight into the evolutionary path of AGN. Furthermore, the upcoming Athena observatory (Nandra et al. 2013) extends the wind studies to a larger population of AGN, from different types and ages, which is key for understanding the role and impact of AGN winds in galaxy evolution.

\section{Conclusions}

In this paper we have carried out broad-band continuum modelling spanning far-IR to hard X-rays, combined with highresolution X-ray and IR spectroscopy, to investigate the nature and origin of dust in IC 4329A. From the findings of our investigation we conclude the following.

1. There are two distinct components of dust in IC 4329A: an ISM dust lane component, and a nuclear component. The nuclear dust component originates from the AGN torus and its associated wind. Dust in the AGN torus is seen through IR emission, while the dust in the torus wind is detected through reddening and X-ray absorption in our line of sight.

2. The AGN wind in IC 4329A consists of three ionisation components. They have moderate outflow velocities: -340 to $-440 \mathrm{~km} \mathrm{~s}^{-1}$ for two of the components, while the other is consistent with zero outflow velocity.

3. According to our variability analysis, the two lowest ionisation components of the AGN wind show no long-term changes between historical and new observations ( $\sim 14$ years apart), while the highest component shows changes in $N_{\mathrm{H}}$ and ionisation parameter $\xi$. From the recombination timescale analysis, we derive limits on the distance of the wind components from the central engine. The two lowest ionisation components are at $r>350 \mathrm{pc}$, and $r>83 \mathrm{pc}$, while the highest ionisation component is at $r<93 \mathrm{pc}$.
4. The total internal reddening in IC $4329 \mathrm{~A}$ is $E(B-V) \approx 1.0$. The reddening is most consistent with a grey (flat) extinction law.

5. From high-resolution X-ray spectroscopy of dust in IC 4329A we derive the total depletion factors from gas into dust for $\mathrm{O}, \mathrm{Si}$, and $\mathrm{Fe}$. They correspond to $17-23 \%$ for $\mathrm{O}$, $70-100 \%$ for $\mathrm{Si}$, and $77-98 \%$ for $\mathrm{Fe}$.

6. The dust grains associated to the AGN torus and its wind in IC 4329A are likely larger than the Milky Way ISM dust, and are in a porous composite form, containing amorphous silicate with iron and oxygen.

7. From on our modelling of the continuum from far-infrared to X-rays, we derive a bolometric luminosity of about $2.5 \times 10^{45} \mathrm{erg} \mathrm{s}^{-1}$ for IC 4329A, which corresponds to 10 $20 \%$ of the Eddington luminosity.

Acknowledgements. M.M. and E.C. are supported by the Netherlands Organisation for Scientific Research (NWO) through The Innovational Research Incentives Scheme Vidi grant 639.042.525. This research has made use of data obtained from the Chandra Data Archive, and software provided by the Chandra X-ray Center (CXC). This work made use of data supplied by the UK Swift Science Data Centre at the University of Leicester. This work is based on observations made with the NASA/ESA Hubble Space Telescope, obtained from the data archive at the Space Telescope Science Institute. This research has made use of the NASA/IPAC Infrared Science Archive, which is operated by the Jet Propulsion Laboratory, California Institute of Technology, under contract with the National Aeronautics and Space Administration. We thank Fred Lahuis for help with the reduction of the Spitzer IRS spectra, and Michiel Min for useful discussions. We thank the anonymous referee for the useful comments.

\section{References}

Alonso-Herrero, A., Ramos Almeida, C., Mason, R., et al. 2011, ApJ, 736, 82 Antonucci, R. 1993, ARA\&A, 31, 473

Antonucci, R. R. J., \& Miller, J. S. 1985, ApJ, 297, 621

Baker, J. G., \& Menzel, D. H. 1938, ApJ, 88, 52

Behar, E., Sako, M., \& Kahn, S. M. 2001, ApJ, 563, 497

Bentz, M. C., Denney, K. D., Grier, C. J., et al. 2013, ApJ, 767, 149

Bohlin, R. C., Savage, B. D., \& Drake, J. F. 1978, ApJ, 224, 132

Brenneman, L. W., Madejski, G., Fuerst, F., et al. 2014, ApJ, 788, 61

Canizares, C. R., Davis, J. E., Dewey, D., et al. 2005, PASP, 117, 1144

Cappi, M., Mihara, T., Matsuoka, M., et al. 1996, ApJ, 458, 149

Cardelli, J. A., Clayton, G. C., \& Mathis, J. S. 1989, ApJ, 345, 245

Costantini, E., Pinto, C., Kaastra, J. S., et al. 2012, A\&A, 539, A32

Crenshaw, D. M., \& Kraemer, S. B. 2001, ApJ, 562, L29

Crenshaw, D. M., Kraemer, S. B., Bruhweiler, F. C., \& Ruiz, J. R. 2001, ApJ, 555,633

Czerny, B., Li, J., Loska, Z., \& Szczerba, R. 2004, MNRAS, 348, L54

de La Calle Pérez, I., Longinotti, A. L., Guainazzi, M., et al. 2010, A\&A, 524, A50

de Vaucouleurs, G., de Vaucouleurs, A., Corwin, Jr., H.G., et al. 1991, Third Reference Catalogue of Bright Galaxies (New York: Springer)

den Herder, J. W., Brinkman, A. C., Kahn, S. M., et al. 2001, A\&A, 365, L7

Deo, R. P., Richards, G. T., Crenshaw, D. M., \& Kraemer, S. B. 2009, ApJ, 705, 14

Disney, M. J. 1973, ApJ, 181, L55

Done, C., Davis, S. W., Jin, C., Blaes, O., \& Ward, M. 2012, MNRAS, 420, 1848

Dorodnitsyn, A., Kallman, T., \& Proga, D. 2008, ApJ, 675, L5

Dorodnitsyn, A., Bisnovatyi-Kogan, G. S., \& Kallman, T. 2011, ApJ, 741, 29

Ferrarese, L., \& Merritt, D. 2000, ApJ, 539, L9

Fruscione, A., McDowell, J. C., Allen, G. E., et al. 2006, in Proc. SPIE, 6270, $62701 \mathrm{~V}$

Fukumura, K., Kazanas, D., Contopoulos, I., \& Behar, E. 2010, ApJ, 715, 636

Gallimore, J. F., Yzaguirre, A., Jakoboski, J., et al. 2010, ApJS, 187, 172

Gaskell, C. M. 2017, MNRAS, 467, 226

Gaskell, C. M., \& Benker, A. J. 2007, ArXiv e-prints [arXiv: 0711. 1013]

Gaskell, C. M., Goosmann, R. W., Antonucci, R. R. J., \& Whysong, D. H. 2004, ApJ, 616, 147

Gorenstein, P. 1975, ApJ, 198, 95

Güver, T., \& Özel, F. 2009, MNRAS, 400, 2050

Hatziminaoglou, E., Hernán-Caballero, A., Feltre, A., \& Piñol Ferrer, N. 2015, ApJ, 803, 110

Henning, T. 2010, ARA\&A, 48, 21 
Hernán-Caballero, A., Hatziminaoglou, E., Alonso-Herrero, A., \& Mateos, S. 2016, MNRAS, 463, 2064

Hopkins, P. F., Strauss, M. A., Hall, P. B., et al. 2004, AJ, 128, 1112

Jenkins, E. B. 2009, ApJ, 700, 1299

Kaastra, J. S., Mewe, R., \& Nieuwenhuijzen, H. 1996, in UV and X-ray Spectroscopy of Astrophysical and Laboratory Plasmas, eds. K. Yamashita, \& T. Watanabe, 411

Kaastra, J. S., Detmers, R. G., Mehdipour, M., et al. 2012, A\&A, 539, A117

Kalberla, P. M. W., Burton, W. B., Hartmann, D., et al. 2005, A\&A, 440, 775

Kinney, A. L., Calzetti, D., Bohlin, R. C., et al. 1996, ApJ, 467, 38

Kollatschny, W., \& Fricke, K. J. 1989, A\&A, 219, 34

Komossa, S., \& Fink, H. 1997, A\&A, 322, 719

Krolik, J. H., \& Kriss, G. A. 2001, ApJ, 561, 684

Krolik, J. H., McKee, C. F., \& Tarter, C. B. 1981, ApJ, 249, 422

Kubota, A., \& Done, C. 2018, MNRAS, 480, 1247

Lee, J. C., Ogle, P. M., Canizares, C. R., et al. 2001, ApJ, 554, L13

Lee, J. C., Xiang, J., Ravel, B., Kortright, J., \& Flanagan, K. 2009, ApJ, 702, 970

Lee, J. C., Kriss, G. A., Chakravorty, S., et al. 2013, MNRAS, 430, 2650

Li, A. 2007, in The Central Engine of Active Galactic Nuclei, eds. L. C. Ho, \& J.-W. Wang, ASP Conf. Ser., 373, 561

Li, M. P., Shi, Q. J., \& Li, A. 2008, MNRAS, 391, L49

Lodders, K., Palme, H., \& Gail, H. P. 2009, Landolt Börnstein, 44

Magdziarz, P., \& Zdziarski, A. A. 1995, MNRAS, 273, 837

Magdziarz, P., Blaes, O. M., Zdziarski, A. A., Johnson, W. N., \& Smith, D. A. 1998, MNRAS, 301, 179

Maiolino, R., Marconi, A., Salvati, M., et al. 2001, A\&A, 365, 28

Malkan, M. A., Jensen, L. D., Rodriguez, D. R., Spinoglio, L., \& Rush, B. 2017, ApJ, 846, 102

Markowitz, A. 2009, ApJ, 698, 1740

Martin, P. G., Stockman, H. S., Angel, J. R. P., Maza, J., \& Beaver, E. A. 1982, ApJ, 255, 65

Marziani, P., Calvani, M., \& Sulentic, J. W. 1992, ApJ, 393, 658

Mason, R. E. 2015, Planet. Space Sci., 116, 97

Mason, K. O., Breeveld, A., Much, R., et al. 2001, A\&A, 365, L36

McHardy, I. M., Koerding, E., Knigge, C., Uttley, P., \& Fender, R. P. 2006, Nature, 444, 730

Mehdipour, M., Branduardi-Raymont, G., Kaastra, J. S., et al. 2011, A\&A, 534, A39

Mehdipour, M., Branduardi-Raymont, G., \& Page, M. J. 2012, A\&A, 542, A30

Mehdipour, M., Kaastra, J. S., Kriss, G. A., et al. 2015, A\&A, 575, A22

Mehdipour, M., Kaastra, J. S., \& Kallman, T. 2016, A\&A, 596, A65

Mehdipour, M., Kaastra, J. S., Costantini, E., et al. 2018, A\&A, 615, A72

Meléndez, M., Mushotzky, R. F., Shimizu, T. T., Barger, A. J., \& Cowie, L. L. 2014, ApJ, 794, 152

Moshir, M., et al. 1990, in IRAS Faint Source Catalogue, version 2.0

Nandra, K., Barret, D., Barcons, X., et al. 2013, ArXiv e-prints [arXiv: 1306.2307]

O’Donnell, J. E. 1994, ApJ, 422, 158
Oppenheimer, B. D., \& Davé, R. 2006, MNRAS, 373, 1265

Osterbrock, D. E., \& Ferland, G. J., 2006, Astrophysics of Gaseous Nebulae and Active Galactic Nuclei (Mill Valley, CA: University Science Books)

Perola, G. C., Matt, G., Cappi, M., et al. 1999, A\&A, 351, 937

Peterson, B. M., Ferrarese, L., Gilbert, K. M., et al. 2004, ApJ, 613, 682

Petrucci, P.-O., Paltani, S., Malzac, J., et al. 2013, A\&A, 549, A73

Pier, E. A., \& Krolik, J. H. 1992, ApJ, 401, 99

Pier, E. A., \& Krolik, J. H. 1993, ApJ, 418, 673

Pinto, C., Kaastra, J. S., Costantini, E., \& Verbunt, F. 2010, A\&A, 521, A79

Porquet, D., Reeves, J. N., Matt, G., et al. 2018, A\&A, 609, A42

Predehl, P., \& Schmitt, J. H. M. M. 1995, A\&A, 293, 889

Proga, D., \& Kallman, T. R. 2004, ApJ, 616, 688

Reynolds, C. S., Ward, M. J., Fabian, A. C., \& Celotti, A. 1997, MNRAS, 291, 403

Rogantini, D., Costantini, E., Zeegers, S. T., et al. 2018, A\&A, 609, A22

Roming, P. W. A., Kennedy, T. E., Mason, K. O., et al. 2005, Space Sci. Rev., 120,95

Schlafly, E. F., \& Finkbeiner, D. P. 2011, ApJ, 737, 103

Shimizu, T. T., Meléndez, M., Mushotzky, R. F., et al. 2016, MNRAS, 456, 3335

Siebenmorgen, R., Haas, M., Krügel, E., \& Schulz, B. 2005, A\&A, 436, L5

Silk, J., \& Rees, M. J. 1998, A\&A, 331, L1

Smith, R. K., Abraham, M. H., Allured, R., et al. 2016, in Proc. SPIE, 9905, 99054M

Steenbrugge, K. C., Kaastra, J. S., Sako, M., et al. 2005, A\&A, 432, 453

Strüder, L., Briel, U., Dennerl, K., et al. 2001, A\&A, 365, L18

Sturm, E., Schweitzer, M., Lutz, D., et al. 2005, ApJ, 629, L21

Tarter, C. B., Tucker, W. H., \& Salpeter, E. E. 1969, ApJ, 156, 943

Tristram, K. R. W., Raban, D., Meisenheimer, K., et al. 2009, A\&A, 502, 67

Tristram, K. R. W., Burtscher, L., Jaffe, W., et al. 2014, A\&A, 563, A82

Urry, C. M., \& Padovani, P. 1995, PASP, 107, 803

Vasudevan, R. V., Fabian, A. C., Gandhi, P., Winter, L. M., \& Mushotzky, R. F. 2010, MNRAS, 402, 1081

Véron-Cetty, M.-P., \& Véron, P. 2006, A\&A, 455, 773

Willingale, R., Starling, R. L. C., Beardmore, A. P., Tanvir, N. R., \& O'Brien, P. T. 2013, MNRAS, 431, 394

Willmer, C. N. A., Focardi, P., Chan, R., Pellegrini, P. S., \& da Costa, N. L. 1991, AJ, 101, 57

Wilson, A. S., \& Penston, M. V. 1979, ApJ, 232, 389

Winge, C., Peterson, B. M., Pastoriza, M. G., \& Storchi-Bergmann, T. 1996, ApJ, 469,648

Wolstencroft, R. D., Done, C. J., Scarrott, S. M., \& Scarrott, R. M. J. 1995, MNRAS, 276, 460

Xie, Y., Li, A., \& Hao, L. 2017, ApJS, 228, 6

Zafar, T., Møller, P., Watson, D., et al. 2015, A\&A, 584, A100

Zeegers, S. T., Costantini, E., de Vries, C. P., et al. 2017, A\&A, 599, A117

Zycki, P. T., \& Czerny, B. 1994, MNRAS, 266, 653

Zycki, P. T., Done, C., \& Smith, D. A. 1999, MNRAS, 305, 231 